DOI - https://doi.org/10.5965/2316796309172020034

\title{
Eletromiografia em ergonomia no Brasil: Estado da arte. Uma revisão sistemática de literatura
}

\section{Eletromiography in ergonomics in Brazil: State of art. A systematic review.}

Lincoln Silva $^{1}$

Eugenio Andrés Díaz Merino ${ }^{2}$ 


\section{Resumo}

A eletromiografia (EMG) é um método quantitativo reconhecidamente útil na avaliação ergonômica. Contudo, não há estudos que demonstrem como este método tem sido utilizado em ergonomia no Brasil. O objetivo deste estudo é verificar em que casos a EMG está sendo em ergonomia no Brasil, por meio de uma revisão sistemática de literatura. Foram selecionados 41 estudos. O setor industrial, serviço de escritório com uso de computador e tecnologia assistiva foram os temas mais abordados. Os músculos mais acessados se relacionam aos membros superiores, cervical e coluna se o uso de questionários, cinemetria se o uso de ques
dinamometria.

\section{Palavras-chave:}

ergonomia, método quantitativo, Brasil.

\section{Abstract}

Electromyography (EMG) is a quantitative useful method in ergonomic assessment. However, there are no studies demonstrating how this method has been used in ergonomics in Brazil. The objective of this study is to verify in which cases the EMG is being used and the adopted procedures in ergonomics in Brazil through a systematic literature review. Forty-one studies were selected. The industrial sector, office service with use of computer and assistive technology were the most approached subjects. The most accessed muscles relate to the upper limbs, cervical and lumbar spine. We highlight che use of questionnaires, cinemetry and dynamometry in conjunction with EMG.

Key-words: electromyography, ergonomics, quantitative method, Brazil.
1 Doutorando em Engenharia, UFSC (lincolnsilvafisio@gmail.com)

2 Doutor em Engenharia, UFSC (eugenio.merino@ufsc.br)

\section{INTRODUÇÃO}

A ergonomia é a disciplina científica que estuda as interações entre os seres humanos e outros elementos de um sistema (IEA, 2000). Quanto aos objetivos, pode estar centrada nas organizações, relacionando-se ao desempenho, confiabilidade, durabilidade de um determinado produto, ou à pessoa, atuando no seu bem-estar físico, emocional e operacional (FALZON, 2007).

A preocupação com o aspecto físico da relação do ser humano com os elementos de um sistema tem sido um tema relevante desde os primeiros estudos em ergonomia, que buscavam melhorar a interação homem-máquina, ao final da segunda guerra mundial (IIDA e GUIMARÃES, 2016). Assim, com o desenvolvimento deste campo de estudo, aprofundou-se o conhecimento relacionado à anatomia, antropometria, fisiologia e biomecânica, pois tais características podem interferir de forma direta no meio laboral (FALZON, 2007).

No tocante às características físicas e cognitivas do indivíduo, pode haver - surgimento da fadiga. Esta pode ser conceituada como uma vulnerabilidade aguda em que o organismo necessita de maior esforço para manter a performance anteriormente alcançada (ENOKA e STUART, 1992) e está relacionada primeiramente às características fisiológicas ligadas ao tempo de realização e intensidade do trabalho (IIDA e GUIMARÃES, 2016).

Surge desta forma, a necessidade de quantificar as características biomecânicas e fisiológicas do homem em seu contexto de trabalho. Esta forma de pesquisar passa por métodos quantitativos de análise e pelo estudo de populações maiores que executam uma mesma atividade, tal como sugerido por Fathallah (2008), para compreensão de distúrbios musculoesqueléticos relacionados ao trabalho no meio rural. Contudo, em estudos relacionados à ergonomia física, as técnicas utilizadas para mensurar esforços físicos, desconforto ou dor, riscos de Distúrbios Osteomusculares Relacionados ao Trabalho (DORT) e manuseio de carga possuem abordagem qualitativa, baseada em instrumentos validados, tais como a Escala de Borg, Questionário Nórdico e os checklists ergonômicos (IIDA e GUIMARÃES, 2016).

Com intuito de quantificar os aspectos fisiológicos e biomecânicos, a EMG tem sido um método amplamente utilizado em estudosergonômicos (KUMAR e MITAL, 1996). A EMG, por ser capaz de reconhecer a atividade muscular em tempo real, e relacionarse com as características fisiológicas das fibras musculares, vem sendo considerada uma ferramenta útil nas avaliações ergonômicas (MARRAS, 1990). Contudo, há grande variação de aplicações e protocolos utilizados neste método e não há estudos que abordem como e em quais situações este método tem sido utilizado nas pesquisas relacionadas à ergonomia no Brasil. Sendo assim, este estudo tem o objetivo de verificar em que casos a EMG está sendo utilizada e os procedimentos adotados nas pesquisas relacionadas à ergonomia no Brasil por meio de uma revisão sistemática de literatura.

\subsection{REFERENCIAL TEÓRICO}

Para um melhor entendimento do tema abordado, será apresentada uma breve síntese de informações relativas à EMG e sua ligação com a ergonomia. 


\subsubsection{EMG}

O deslocamento dos filamentos de actina e miosina no interior de um arranjo muscular pode provocar a produção de força na contração de um músculo. Este deslizamento dos filamentos é resultado da variação do potencial elétrico na membrana celular. O registro desta atividade elétrica, gerada pelo potencial de ação, no interior da célula muscular é a EMG (KUMAR e MITAL, 1996).

Para entender o mecanismo de contração muscular, é necessária a compreensão de que a produção de força é resultado da interação do músculo com o sistema nervoso central e periférico, no qual o motoneurônio-alfa, em conjunto com as fibras musculares que são conectadas a ele, formam a Unidade Motora (UM) que será responsável pela comunicação do músculo com o sistema nervoso (MERLETTI, 2004), (figura 1).

Figura 1: Caminho neural para produção da contração musculoesquelética.



Fonte: Autores, 2018 (Adaptado de Merletti, 2004, p. 3)

O sinal eletromiográfico pode ser considerado como um indicador de força, pois aborda o nível de excitação neural musculoesquelética (LINDSTROM; KADERFORS; PETERSEN, 1977). Outros aspectos importantes que podem ser visualizados pela EMG são: a duração de uma contração muscular, a participação dos grupos musculares em um determinado movimento, a quantidade de fibras musculares usadas e a frequência de ativação das UMs (NODA; MARCHETTI; VILELA, 2014).

Por outro lado, a análise eletromiográfica possibilita uma visão setorizada do uso da musculatura, pois em grupos musculares muito extensos não há possibilidade de cobertura de toda a superfície muscular com o uso dos eletrodos de superfície. Sendo assim, em um determinado movimento, a força poderá se manter, mesmo havendo a queda do padrão do sinal eletromiográfico inicial, pois neste caso, poderão estar sendo ativadas outras UMs distantes das captadas pelos eletrodos superficiais (DE LUCA, 1997; SIEGLER et al.,1985).

Há ainda outros fatores que podem interferir nas características do sinal captado, tais como: a bioimpedância dos tecidos subcutâneos que estão entre os eletrodos e o músculo avaliado, que pode variar de acordo com a localização e composição corporal de cada indivíduo. Desta forma, não é possível comparar valores eletromiográficos absolutos entre indivíduos distintos. Porém, se o sinal for normalizado previamente, com base em um sinal mioelétrico de referência, os valores poderão ser expressos em percentuais, podendo ser usados em estudos comparativos (BIGLAND-RITCHIE, 1981; DE LUCA, 1997).

Em relação à normalização do sinal, há grande variação nos métodos e uso de referenciais (BURDEN, 2010). Contudo, a forma de normalização do sinal comumente usada é com base no valor máximo ou média dos valores máximos de uma contração muscular, para o movimento que será analisado (MATHIASSEN et al, 1995; BASMAJIAN; DE LUCA, 1985).

\subsubsection{EMG em ergonomia}

O registro da atividade elétrica muscular em situações laborais tem aproximadamente 67 anos. O primeiro estudo publicado foi conduzido por Lundervold, neurofisiologista norueguês, que avaliou 47 indivíduos que trabalhavam sentados na atividade de datilografia. Neste estudo foram avaliados os músculos trapézio grande dorsal, e músculos da região lombo sacral. Com base na interpretação do sinal eletromiográfico, o pesquisador afirmou que a posição em que há menor esforço muscular para realizar a datilografia era com os pés bem apoiados no chão, joelhos bem posicionados e o tronco sendo suportado pela região lombar (LUNDERVOLD, 1951)

O registro da atividade elétrica muscular é um bom mecanismo para quantificar o tipo e o grau de solicitação muscular em uma atividade de trabalho, postura ou interação do ser humano com determinado elemento (IIDA e GUIMARÃES, 2016). Segundo Kumar e Mital (1996), a EMG pode ser considerada uma ferramenta poderosa na ergonomia, sendo crucial na tomada de decisão do ergonomista podendo trazer três formas de apresentação dos dados: quantitativa, semi-quantitativa e qualitativa. Contudo, a sofisticação do método traz cuidados especiais para que as respostas sejam válidas, tais como: a escolha apropriada do músculo, preparação do local para colocação correta dos eletrodos, gravação adequada dos dados e interpretação precisa.

Em relação às aplicações da EMG na ergonomia, o método pode apresentar as respostas a seguir (KUMAR e MITAL, 1996):

a) Se há atividade muscular em uma determinada tarefa;

b) Quando ocorre o acionamento muscular?

c) Qual é interação entre os músculos de interesse na produção do movimento?

d) Houve aquisição de habilidades, com base no padrão de ativação muscular?

e) Houve maior atividade eletromiográfica relacionada ao estresse da atividade de trabalho, medida por um ou mais dos seguintes itens semi-quantitativos variáveis?

(i) Magnitude de sinais brutos;

(ii) Número de cruzamento zero;

(iii) Número e / ou magnitude de picos;

f) A magnitude da atividade eletromiográfica baseada em fatores quantitativos, segundo as medidas listadas abaixo:

(i) tensão $(\mu \mathrm{V} ; \mathrm{mV})$;

(ii) Retificação de ondas e detecção de envelope;

(iii) Integral do sinal;

(iv) RMS (Root Mean Square); 
(v) Normalização e cálculo de força por meio do sinal eletromiográfico;

g) Fadiga muscular.

Recentemente, a EMG tem sido utilizada em diversas áreas da ergonomia. Como exemplo, pode-se citar a aplicação na indústria, por meio dos estudos no setor automotivo (GRAHAM, 2009); na agricultura, com estudo da relação do homem com as máquinas agrícolas (KIM, 2018) e em atividades de grande esforço físico, como a retirada de raízes de mandioca do solo (MERINO, 2017) e na tecnologia assistiva, colaborando com o entendimento da interação do homem com as órteses, próteses e cadeiras de rodas (DA SILVA et al, 2017).

\section{PROCEDIMENTOS METODOLÓGICOS}

Trata-se de uma revisão sistemática de literatura, com base no método proposto por Sampaio e Mancini (2007), que propõe 5 etapas de desenvolvimento da revisão (Figura 2).

Figura 2: Etapas para desenvolvimento de uma revisão sistemática de literatura



Fonte: Autores, 2018

Conforme o método proposto, foi definida a pergunta de pesquisa: De que forma e em quais situações a EMG tem sido utilizada nas pesquisas relacionadas à ergonomia no Brasil? Para buscar as evidências, foram consultadas as bases de dados: Scielo, Google Scholar, Catálogo de Teses e Dissertações da Capes (CTDC) e Biblioteca Digital Brasileira de Teses e Dissertações (BDTD). Além destas bases, foi realizada pesquisa nos anais de eventos nacionais relacionados à ergonomia: Encontro Nacional de Engenharia de Produção (ENEGEP); Simpósio de Engenharia Produção (SIMPEP); Congresso Brasileiro de Ergonomia (ABERGO); Congresso Internacional de Ergonomia e Usabilidade de Interfaces Humano Tecnológica: Produto, Informações Ambientes Construídos e Transporte (Ergodesign). Para consulta dos artigos nos bancos de dados Scielo e Google Scholar foram utilizados os termos "eletromiografia" e "ergonomia". Quanto às teses, dissertações e estudos publicados em anais de eventos, utilizou-se o termo "eletromiografia". Os artigos obtidos foram selecionados considerando sua relação com o tema pesquisado, baseados na análise do título, palavras-chave, resumo e metodologia. Após triagem inicial, foram lidos os títulos e selecionados os estudos para leitura do resumo e procedimentos metodológicos. Os estudos escolhidos foram lidos integralmente para obtenção dos dados referentes à pergunta de pesquisa. Foram avaliados apenas os estudos escritos no idioma português e inglês, publicados em periódicos nacionais, com data de publicação compreendida entre os anos 2009 e 2018.

\section{RESULTADOS}

Os resultados serão divididos em três blocos de informações: i) o primeiro apresentará as informações referentes artigos encontrados em periódicos nacionais; ii) o segundo, será referente às dissertações e teses; iii) o terceiro, referente aos artigos de eventos. Por fim, será apresentada uma síntese de informações referente aos músculos acessados, temas de estudo e procedimentos metodológicos.

\section{i)Artigos Publicados em Periódicos}

A busca de artigos publicados em periódicos nacionais ocorreu no dia 07 de agosto de 2018. Foram selecionados 12 artigos em periódicos nacionais, que atenderam aos critérios de escolha (Figura 3). Esses são apresentados na tabela 1.

Foi observado, quanto aos temas de estudo, que o trabalho de escritório com uso de computadores apresentou o maior número estudos, seguido pelo setor industrial, tecnologia assistiva, serviço odontológico e militar, respectivamente. Contudo, não foram encontrados estudos ergonômicos, com uso de EMG, no setor rural e de esportes.

Figura 3: Percurso metodológico para seleção de artigos publicados em periódicos nacionais.



Fonte: Autores, 2018

No que tange aos objetivos dos artigos de periódicos selecionados, esses concentraram-se em verificar ou avaliar a atividade eletromiográfica em meio ao trabalho, nas quais a resposta eletromiográfica mais abordada foi a RMS. Para atingir os objetivos de pesquisa, algumas ferramentas foram empregadas em conjunto à EMG, destacando-se nos métodos qualitativos, o uso de questionários e escalas ligadas à ergonomia, e nos métodos quantitativos, a cinemetria, dinamometria, eletrogoniometria e a inclinometria. Não foram encontrados estudos correlacionais entre os dados qualitativos e quantitativos, bem como entre os dedos quantitativos.

No tocante à contribuição da EMG nos estudos ergonômicos publicados em periódicos nacionais, foram demonstrados dados relacionados à fadiga muscular, ao uso de diferentes grupos musculares em meio às atividades de trabalho e comparações de atividades mioelétricas antes e depois de intervenções ergonômicas.

Os músculos mais acessados nos artigos foram: trapézio, deltoide, bíceps braquial, extensores e flexores do punho.

Foi observada ainda a classificação dos periódicos, nos quais foram publicados os artigos selecionados, segundo a classificação de periódicos do quadriênio (2013-2016) 
da Coordenação de Aperfeiçoamento de Pessoal de Nível Superior (CAPES), relacionada à área de Engenharias III, devido a ergonomia estar dentro deste grupo. Dentre os 12 artigos, 1 foi publicado em periódico classificado como B1, 2 em B3, 1 em B4, 3 em B5 e 5 artigos publicados em periódicos nacionais não classificados pela CAPES.

\section{ii) Teses e Dissertações}

A busca de teses e dissertações ocorreu do dia 6 ao dia 21 de Junho de 2018. Foram encontrados, no Catálogo de Teses e Dissertações da Capes (CTDC) e na Biblioteca Digital Brasileira de Teses e Dissertações (BDTD), 1707 estudos relacionados à EMG, realizados entre 2009 e 2018. Destes 1707, 581 e 1126 correspondem ao número de teses e dissertações, respectivamente. Após leitura dos títulos e retirada dos duplicados, foram selecionados 59 estudos (Figura 4). Por fim, após leitura dos resumo e procedimentos metodológicos, foram escolhidos 28 estudos e lidos integralmente, sendo apresentados na tabela 2 .

Figura 4: Percurso metodológico para seleção das teses e dissertações.

\section{Teses e Dissertações}



Fonte: Autores, 2018

Pôde-se constatar, em relação aos temas de estudo, nos quais foi aplicada a EMG nas teses e dissertações, que o setor industrial apresentou maior número, sendo seguido pelo trabalho de escritório com uso de computadores, tecnologia assistiva, setor aeronáutico militar, serviço odontológico, trabalho rural e ergonomia esportiva, respectivamente.

Nestas pesquisas, o principal objetivo de estudo, relacionado à EMG, foi avaliar ou analisar a atividade muscular relacionada ao trabalho, com predominância da análise da RMS. Com intuito de alcançar esses objetivos, foram empregadas ferramentas em conjunto à EMG, sobressaindo como método qualitativo, o uso de questionários e escalas ergonômicas, e em relação aos métodos quantitativos, a cinemetria, dinamometria, antropometria, biofotogrametria, eletrogoniometria e inclinometria. Não foram encontrados estudos correlacionais entre os dados qualitativos e a atividade elétrica muscular, caracterizando a avaliação individualizada de cada resposta apresentada pelas ferramentas, não sendo exploradas as correlações e interdependências entre os achados.

Quanto à contribuição da EMG nos estudos ergonômicos de teses e dissertações selecionados, foram observados dados relacionados à fadiga muscular em meio a situações reais de trabalho, distinção de grupos musculares mais utilizados e comparações da atividade muscular antes e após adaptações ergonômicas. Os músculos mais acessados nas pesquisas foram respectivamente: trapézio, deltoide, bíceps braquial, tríceps braquial, flexores e extensores do punho e a musculatura eretora da coluna vertebral.

$\mathrm{Em}$ relação ao local onde foram realizadas as pesquisas, observa-se que entre 2009 e 2018, apenas as regiões sudeste e sul do Brasil apresentam estudos de teses e dissertações, com uso de EMG, na ergonomia. Contudo, dos 28 estudos selecionados, 22 foram orientados em universidades pertencentes ao estado de São Paulo, com destaque para UNESP (7), UFSCAR (6) e USP(5), 2 em Minas Gerais, na Universidade Federal de Viçosa, 3 em universidades do Paraná e 1 no estado do Rio Grande do Sul.

\section{iii) Artigos de Eventos}

A busca de artigos publicados em eventos ocorreu entre os dias 6 e 10 de Agosto de 2018. Foi encontrado apenas um estudo (Figura 5) que utilizou a EMG com intuito de fazer uma análise biomecânica e ergonômica da atividade de arranque manual de raízes de mandioca. Este estudo abordou um tema relacionado ao trabalho no meio rural e gerou informações relacionadas à fadiga e força da musculatura extensora lombar em meio a uma atividade manual de grande esforço (Tabela 3).

$$
\text { Figura 5: Percurso metodológico para seleção dos artigos de eventos. }
$$


Fonte: Autores, 2018

Emsíntese, conformefigura 6, observa-se que os estudosanalisadosapresentaram considerável variação de grupos musculares acessados, porém com predominância nos músculos relacionados aos membros superiores e região cervical. Os métodos quantitativos mais utilizados foram os questionários. 
Figura 6: Síntese relacionada à EMG em ergonomia no Brasil (2009-2018)



MÉTODOS



Músculos


Fonte: Autores, 2018

Enquanto que nos métodos quantitativos, a cinemetria, dinamometria e inclinometria foram os mais utilizados em conjunto com a EMG. Em relação às áreas de pesquisa, destaca-se o setor industrial, o trabalho de escritório com uso de computador e os estudos em tecnologias assistivas. As pesquisas no setor de serviços odontológicos e aéreo militar apresentaram o mesmo número de trabalhos. Os setores rural e esporte apresentaram apenas 3 e 1 estudos, respectivamente.

\section{CONCLUSÕES}

A adoção de métodos quantitativos na avaliação ergonômica pode trazer clareza à tomada de decisão do ergonomista. A EMG é um método quantitativo que tem sido utilizado em avaliações ergonômicas por aproximadamente 67 anos. Este método é reconhecido como útil e importante aliado na ação ergonômica. Sendo assim, o objetivo deste estudo foi verificar em que casos a EMG está sendo utilizada e os procedimentos adotados nas pesquisas relacionadas à ergonomia no Brasil por meio de uma revisão sistemática de literatura.

Nesta revisão, foi possível observar maior concentração de estudos relacionados ao setor industrial (31,7\%) e ao trabalho de escritório ligado ao uso de computadores (19,5\%). Também foram encontrados estudos em tecnologia assistiva $(14,6 \%)$, serviço aéreo militar (12,2\%), odontologia (12,2\%), produção rural $(7,3 \%)$ e ergonomia esportiva
$(2,4 \%)$. As áreas rural e ergonomia no esporte apresentaram o menor número de estudos com EMG em ergonomia, com 3 e 1 estudos, respectivamente. Considerando a importância nacional do setor rural sendo o Brasil um dos países que possui maior área de cultivo agrícola, faz-se necessário estudos mais aprofundados relacionados à produção rural e aos riscos à saúde dos trabalhadores desse setor. Neste sentido, a EMG pode surgir como uma oportunidade de aprimoramento da análise ergonômica nesta área. Esta mesma oportunidade de aperfeiçoamento poderá ser estendida ao desenvolvimento ergonômico de produtos esportivos.

Outro ponto demostrado neste estudo, foi a condensação de pesquisas em ergonomia, com EMG, na região sudeste $(85,7 \%)$ e sul $(14,3 \%)$. Considera-se que cada região do país possua particularidades relacionadas ao tipo de trabalho, produção e trabalhador. Sendo assim, o uso de métodos quantitativos pode favorecer o acompanhamento e aprimoramento produtivo voltado à singularidade de cada região. Logo, o desenvolvimento da aplicação da EMG em estudos ergonômicos, nas regiões norte, nordeste e centro-oeste poderá corroborar o aprimoramento produtivo, prevenindo a fadiga e lesões relacionadas ao trabalho.

Considera-se ainda, que os estudos de teses e dissertações analisados possuem boa qualidade metodológica, sendo, em muitos casos, encaminhados para publicação em periódicos internacionais, porém esta revisão limitou-se à pesquisa em periódicos nacionais. Em relação aos artigos selecionados, segundo a classificação de periódicos da CAPES para Engenharias III, apenas 1 estudo foi publicado em periódico de estrato B1, sendo que os outros 11 foram publicados em periódicos B3 (2), B4 (1), B5 (3) e sem classificação (5). Contudo, recomenda-se que seja realizado um novo estudo de revisão sistemática de literatura, com intuito de abordar as características metodológicas e a qualidade dos estudos que envolvem a EMG na ergonomia, de grupos brasileiros, publicados em periódicos internacionais.

Com relação à metodologia empregada nas pesquisas analisadas, observase que a resposta eletromiográfica mais acessada foi a RMS e a normalização pela contração voluntária máxima. A Frequência Mediana, considerada como a principal resposta eletromiográfica, relacionada ao desgaste fisiológico muscular e fadiga, foi observada por aproximadamente $10 \%$ dos 41 estudos analisados. Considera-se a RMS como a leitura quantitativa da intensidade de recrutamento de fibras musculares em uma contração muscular. Esta pode ser influenciada pelo acionamento de outras unidades motoras fora do alcance dos eletrodos, sobretudo em músculos maiores, podendo favorecer ao erro na interpretação dos dados.

Com intuito de diminuir este erro, outros métodos de análise ergonômica podem ser aplicados em conjunto com a EMG, ampliando a visão do ergonomista quanto a avaliação que está sendo realizada. Nos estudos analisados, foram utilizadas ferramentas qualitativas e quantitativas em conjunto à EMG, porém não foi encontrado estudos correlacionais entre os dados qualitativos e o sinal eletromiográfico, configurando assim uma oportunidade para o desenvolvimento da pesquisa em ergonomia ligada à EMG.

Outro dado metodológico demonstrado nesta pesquisa foi o grupo muscular acessado. A maior parte dos estudos seguiu as orientações da SENIAM, para preparação da pele e colocação dos eletrodos. Os grupos musculares mais acessados relacionamse à cintura escapular, região cervical, flexores e extensores do punho e dedos das mãos e região lombar. 


\section{AGRADECIMENTOS}

Agradecemos ao Conselho Nacional de Desenvolvimento Científico e Tecnológico (CNPq) e à Coordenação de Aperfeiçoamento de Pessoal de Nível Superior (CAPES) pelo apoio financeiro para realização desta pesquisa, e ao Núcleo de Gestão de Design (NGD) e Laboratório de Design e Usabilidade (LDU) pela disponibilização do laboratório. Também agradecemos a mestranda Júlia Marina da Cunha por ajudar na construção das figuras deste estudo.

\section{REFERÊNCIAS}

ARAÚJO, Nívia Cecília Kruta. Análise ergonômica do trabalho de catadores de materiais recicláveis. São Carlos, 2017. Tese (Doutorado) - Universidade Federal de São Carlos.

BARBIERI, Dechristian França. Estudo de sintomas musculoesqueléticos, fatores de risco e exposição física em trabalhadores de escritório. São Carlos, 2013. Dissertação (Mestrado) - Programa de Pós-graduação em Fisioterapia de Universidade de São Carlos.

BASMAJIAN J. V.; DE LUCA C. J. Muscles Alive. Baltimore - MD:

Williams and Wilkins. 1985.

BIASIBETTI, Eliana et al. Atividade eletromiográfica de trapézio superior com o sem apoio de antebraço durante a digitação. Revista Destaques Acadêmicos, v. 8, n. 3, 2016

BIGLAND-RITCHIE, B. EMG/force relations and fatigue of human voluntary contractions. Exercise Sport and Science Reviews, v.9, p.75-117, 1981.

BURDEN, Adrian. How should we normalize electromyograms obtained from healthy participants? What we have learned from over 25 years of research. Journal of electromyography and kinesiology, v. 20, n. 6, p. 1023-1035, 2010.

COMEL, João Carlos et al. Comparison of the electrical activity in upper trapezius and wrist extensor muscles during two typewriting conditions. Fisioterapia em Movimento, v. 27, n. 2, p. 271-279, 2014.

CUSTÓDIO, Renata Aparecida Ribeiro. Proposta de um novo conceito de mocho odontológico. Guaratinguetá, 2011.Tese (Doutorado) - Universidade Estadual Paulista, Faculdade de Engenharia de Guaratinguetá.

DASILVA, Emília Pio. Distúrbios osteomusculares relacionados aotrabalho deoperadores da colheita florestal mecanizada. Viçosa, 2011.Tese (Doutorado) - Universidade Federal de Viçosa.

DA SILVA, Sara Raquel Martins et al. Correlação entre ativação muscular e níveis de torque propulsor em diferentes tarefas de mobilidade em cadeira de rodas. Human Factors in Design, v. 6, n. 12, p. 002-014, 2017.
DE LUCA, Carlo J. The use of surface electromyography in biomechanics. Journal of applied biomechanics, v. 13, n. 2, p. 135-163, 1997.

ENOKA, ROGER M.; STUART, DOUGLAS G. Neurobiology of muscle fatigue. Journal of applied physiology, v. 72, n. 5, p. 1631-1648, 1992.

ESTEVAM, Dayane de Oliveira et al.Análise eletromiográfica do padrão de contração muscular durante a digitação em computador e em máquinas de escrever. Ciência ET Praxis, v. 5, n. 10, p. 53-56, 2012.

FAGANELLO, Laís Regina. Aspectos ergonômicos do design de calçados com salto para mulheres com hiperidrose plantar. Bauru, 2016. Dissertação (Mestrado) - Universidade Estadual Paulista.

FATHALLAH, F. A.; MILLER, B. J.; MILES, J. A. Low back disorders in agriculture and the role of stooped work: scope, potential interventions, and research needs. Journal of agricultural safety and health, v. 14, n. 2, p. 221-245, 2008

FERRARI, D.; MATTOS, D.; SILVA, L.; MERINO, G.; SANTOS, J.; MERINO, E. A.Análise postural da atividade com roçadeira motorizada utilizando tecnologia de rastreamento de movimentos 3D In: ERGOTRIPDESIGN, , Aveiro, 2017. VI Ergotrip Design. Aveiro: UA Editora, 2017. v.1. p.37 - 37

FERRIGNO, Iracema Serrat Vergotti. Eletromiografia do membro superior no uso do computador: estudo comparativo entre duas ortes de punho. Campinas, 2009. Tese (Doutorado) - Universidade Estadual de Campinas.

GONÇALVES, Josiane Sotrate. Efeito da descarga de peso e da amplitude de movimento de flexão do ombro sobre a ativação muscular em condições estáticas e dos fatores ergonômicos e psicossociais durante atividade simulada de mouse em estudantes universitárias. São Carlos, 2015. Dissertação (Mestrado) - Universidade Federal de São Carlos.

GRAHAM, Ryan B.; AGNEW, Michael J.; STEVENSON, Joan M. Effectiveness of an onbody lifting aid at reducing low back physical demands during an automotive assembly task: Assessment of EMG response and user acceptability. Applied Ergonomics, v. 40, n. 5, p. 936-942, 2009.

IEA- International Ergonomics Association. Definição Internacional de Ergonomia. San Diego, USA: 2000.

IIDA, Itiro; GUIMARÃES, Lia Buarque de Macedo.Ergonomia: Projeto e Produção. São Paulo: Blucher, 2016.

FILUS, Rodrigo. Utilização da imagem infravermelha normalizada para diagnóstico de doenças ocupacionais e correlação com eletromiografia de superfície e ressonância magnética. Curitiba, 2011. Tese (Doutorado) - Universidade Federal do Paraná. 
KIM, Jeong Ho; DENNERLEIN, Jack T.; JOHNSON, Peter W. The effect of a multi-axis suspension on whole body vibration exposures and physical stress in the neck and low back in agricultural tractor applications. Applied ergonomics, v. 68, p. 80-89, 2018.

KOMINO, C.S.M. Análise eletromiográfica da fase inicial da autopropulsão de cadeira de rodas manual. São Carlos, 2017. Dissertação (Mestrado) - Programa de Pós-Graduação Interunidades Bioengenharia - Escola de Engenharia de São Carlos / Faculdade de Medicina de Ribeirão Preto / Instituto de Química de São Carlos da Universidade de São Paulo.

KUMAR, S e MITAL, A. Electromiography in ergonomics. UK: Taylor \& Francis, 1996.

AFETÁ, J. C. et al. Repercussões imediatas da ginástica laboral preparatória na atividade eletromiográfica do músculo deltoide anterior. Motricidade, v. 8, n. Supl. 2, 2012.

LEONEL, Daniel Vilela. Análise ao longo do tempo da postura do profissional endodontista durante o preparo químico-mecânico do sistema de canais radiculares. São Paulo, 2012. Tese (Doutorado) - Universidade de São Paulo.

LINDSTROM, L.; KADERFORS, R.; PETERSEN, I. An electromyographic index for localized muscle fatigue. Journal of Applied Physiology, v.43, 1977. 750-754 p.

LUNDERVOLD, Arne. Electromyographic investigations during sedentary work, especially typewriting. American Journal of Physical Medicine \& Rehabilitation, v. 30, $n$. 5, p. 314, 1951.

MACEDO, Regina Moreira Borges de. Ergonomia aplicada na redução da dor lombar em ciclistas com o suporte da eletromiografia. Curitiba, 2014. Dissertação (Mestrado) - Programa de Pós-Graduação em Engenharia Biomédica, Universidade Tecnológica Federal do Paraná.

MAGALHAES, Marcos Castelo. Avaliação de conforto em assentos aeronáuticos. São José dos Campos, 2009. Dissertação (Mestrado) - Instituto Tecnológico de Aeronáutica.

MARRAS, William S. Industrial electromyography (EMG). International Journal of Industrial Ergonomics, v. 6, n. 1, p. 89-93, 1990.

MATHIASSEN, S.E, WINKEL, J., HAGG, G.M. Normalization of surface EMG amplitude from the upper trapezius muscle in ergonomic studies: a review. J Electromyogr Kinesiol, 5:197-226, 1995.

MENDES, A.A.M.T. Análise epidemiológica e biomecânica de soldadores de uma indústria metalúrgica naval. São Paulo, 2014. Dissertação (Mestrado) - Programa de Mestrado e Doutorado em Fisioterapia da Universidade Cidade de São Paulo. 2014.

MERINO, Eugenio A D; SILVA, Lincoln da ; MATTOS, Diego Luiz de ; GUIMARÃES, Bruno Maia ; BORGES, Noe Gomes ; DOMENECH, Susana Cristina ; MERINO, Giselle Schmidt A
D . Análise ergonômica e biomecânica da colheita da raíz da mandioca: estudo de caso. In: $16^{\circ}$ Ergodesign Congresso Internacional de Ergonomia e Usabilidade de Interfaces Humano Tecnológica, 2017, Santa Catarina. Blucher Design Proceedings, 2016. p. 15561564. (a)

MERLETTI, Roberto; PARKER, Philip A. Electromyography: physiology, engineering, and non-invasive applications. John Wiley \& Sons, 2004.

MELLO, Marlise Capa Verde Almeida de et al. Eletromiografia de superfície da região cervical-contribuição para a saúde muscular. Acta Paul. Enferm.(Online), v. 30, n. 5, p. 512-519, 2017.

MONTEIRO, João André Bernini. Análise da rotação ombro-quadril e co-contração muscular do tronco durante um movimento de rotação axial com manuseio de carga. Bauru, 2015. Dissertação (Mestrado) - Universidade Estadual Paulista.

NETO, Luís Ferreira MONTEIRO. Estresse físico e psicofisiológico na desossa em frigoríficos bovinos e a macro pausa de descanso durante atividade. São José do Rio Preto 2014. Tese (Doutorado) - Faculdade de Medicina de São José do Rio Preto.

NETO , Luis Ferreira Monteiro; MOSCARDINI, Airton Camacho; ALIOTO, Olavo Egídio; BRAZ, Allison Gustavo. Análise dinâmica da fadiga de trabalhadores do setor de desossa de um frigorífico durante a jornada de trabalho. Terapia manual, v. 10, n.49, p. 277-283, 2012.

NODA, Dayna Karina Goberna; MARCHETTI, Paulo Henrique; JUNIOR, Guanis de Barros Vilela. A Eletromiografia de superfície em estudos relativos à produção de força. Revista CPAQV-Centro de Pesquisas Avançadas em Qualidade de Vida, v. 6, n. 3, p. 2, 2014

NOGUEIRA, Helen Cristina. Exposição biomecânica durante o manuseio de caixasem ambiente real e simulado com trabalhadores industriais. São Carlos, 2016. Tese (Doutorado) - Universidade Federal de São Carlos.

OLIVEIRA, Danielle Rodrigues de. Aspectos ergonômicos do levantamento manual de carga em mulheres: relação com equação de NIOSH. Guaratinguetá, 2014.Tese (Doutorado) - Universidade Estadual Paulista.

ONETY, Geraldo Celso da Silva. Análise postural do endodontista na atividade clínica utilizando instrumentação rotatória e manual por meio da cinemetria, eletromiografia de superfície e checklists ergonômicos. São Paulo, 2011.Tese (Doutorado) - Universidade de São Paulo. 2011

ONETY, Geraldo Celso da Silva et al. Analysis of endodontist posture utilizing cinemetry, surface electromyography and ergonomic checklists. Brazilian dental journal, v. 25, n. 6, p. 508-518, 2014.

FALZON, Pierre. Ergonomia. São Paulo: Blucher, 2007.

PINHEIRO, Carina Ferreira. Relação de flexão-relaxamento dos músculos cervicais e dor HFD, v.9, n 17, p. 34-57, junho 2020 
cervical crônica em trabalhadores de escritório usuários de computador. São Paulo, 2015. Tese (Doutorado) - Universidade de São Paulo.

REGIS FILHO, Gilsée Ivan; MICHELS, Glaycon; SELL, Ingeborg. Lesões por esforços repetitivos/distúrbios osteomusculares relacionados ao trabalho de cirurgiõesdentistas: aspectos biomecânicos. Production, v. 19, n. 3, p. 569-580, 2009.

ROCHA, Guilherme Görgen da. Saúde do trabalhador e dor lombar: fatores associados e novas tecnologias analíticas. Santa Cruz do Sul, 2017. Dissertação (Mestrado) Universidade de Santa Cruz do Sul - UNISC.

RODRIGUES, Daniel Érico. Análise comparativa da atividade muscular do flexor superficial dos dedos e força de preensão palmar em atividades laborativas de uma indústria. São Paulo, 2014. Dissertação (Mestrado) - Universidade Estadual Paulista.

RONCATTO, Sílvia Conte et al. Projeto de órteses: definição de requisitos com base no usuário, produto e contexto de uso. Revista Ação Ergonômica, v. 12, n. 2, 2017.

SAMPAIO, Rosana F.; MANCINI, Marisa C. Systematic review studies: a guide for carefu synthesis of the scientific evidence. Brazilian Journal of Physical Therapy, v. 11, n. 1, p. 83-89, 2007.

SANTOS, Michele Caroline dos et al. Análise eletromiográfica da sobrecarga postural causada pelo colete balístico em profissionais de seguranca pública. Curitiba, 2016. Dissertação (Mestrado) - Universidade Tecnológica Federal do Paraná.

SANTOS, Michele Caroline dos; KRUEGER, Eddy; NEVES, Eduardo Borba. Electromyographic analysis of postural overload caused by bulletproof vests on public security professionals. Research on Biomedical Engineering, 2017.

SILVA, Larissa Galvão. Órteses em PVC para membro superior: utilização por terapeutas ocupacionais brasileiros, propriedades físico mecânicas e de toxicidade e desempenhos funcional e mioelétrico. São Carlos, 2013. Dissertação (Mestrado) - Universidade Federal de São Carlos.

SILVA, Luciana Cristina da Cunha Bueno. Avaliação de pegas e sobrecarga dos membros superiores durante o manuseio de caixas: aspectos biomecânicos e perceptuais. São Carlos, 2012. Tese (Doutorado) - Universidade federal de São Carlos.

SILVA, Luciana C. C. B. et al. $30^{\circ}$ de inclinação em alças de caixas plásticas pode reduzi sobrecarga postural e muscular em manuseio de peso. Braz. J. Phys. Ther., São Carlos, v. 17, n. 3, p. 307-318, June 2013

SILVA, Sara Raquel Martins da. Influência do design de cadeira de rodas manual nos aspectos biomecânicos, perceptivos e de desempenho na mobilidade de usuários não usuários. Bauru, 2018. Dissertação (Mestrado) - Universidade Estadual Paulista. Faculdade de Arquitetura Artes e Comunicação.
SHIMANO, Suraya Gomes Novaes. Estudo ergonômico de Pilotos do Esquadrão de Demonstração Aérea Brasileiro. Ribeirão Preto, 2010. Tese (Doutorado) - Faculdade de Medicina de Ribeirão Preto (FMRP/USP).

SIEGLER, S.; HILLSTROM, H. J.; FREEDMAN, W.; MOSKOWITZ, G. Effect of myoeletric signal processing on the relationship between muscle force and processed EMG. American Journal of Physical Medicine, Baltimore, v.64, n.3, , p.130-149, 1985.

VASCONSELOS, G.B.T. Análise ergonômica da fabricação de estruturas de sofá em uma indústria moveleira. Viçosa, 2017. Dissertação (Mestrado) - Universidade Federal de Viçosa. 
Tabela 1: Resumo dos estudos selecionados em periódicos nacionais.

\begin{tabular}{|c|c|c|c|c|c|c|c|}
\hline Autor & Ano & Tisalo & Objetivo & Amostra & Merodo & Contribuilço EMG & $\begin{array}{c}\text { Cuallis } \\
\text { CAPEs } \\
\text { (Engentharias } \\
\text { int) }\end{array}$ \\
\hline  & 2009 &  & 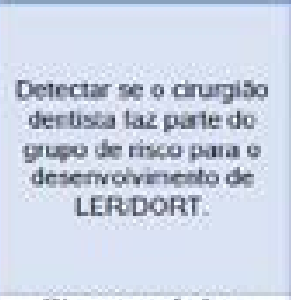 & 4 &  &  & 83 \\
\hline $\begin{array}{l}\text { NETO. L.F. et } \\
\text { al. }\end{array}$ & 2012 &  &  & 18 &  &  & 84 \\
\hline $\begin{array}{l}\text { ESTEVAan, } \\
\text { D.o. at al. }\end{array}$ & 2012 &  &  & 20 &  &  & 0 \\
\hline $\begin{array}{c}\text { LaFETA, J.C. } \\
\text { ot } \pm 1 .\end{array}$ & 2012 &  &  & 19 & EMG &  & 83 \\
\hline & & $\begin{array}{l}\text { músculo dechode } \\
\text { arterior }\end{array}$ &  & & & & \\
\hline $\begin{array}{l}\text { siva } \\
\text { LC.C.B. atal }\end{array}$ & 2013 &  &  & 37 & $\begin{array}{l}\text { Escala Sutipliva de } \\
\text { Agradabidade, } \\
\text { eletogonicmetia } \\
\text { inchinometia. EMG }\end{array}$ &  & B) \\
\hline $\begin{array}{l}\text { CONEL L.C. } \\
\text { ot al. }\end{array}$ & 2014 &  &  & 6 &  &  & 0 \\
\hline $\begin{array}{l}\text { ONETY, a.C.S. } \\
\text { "t at. }\end{array}$ & 2014 &  &  & 18 &  &  & 0 \\
\hline $\begin{array}{c}\text { BIASIBETII, E. } \\
\text { "t al. }\end{array}$ & 2015 & 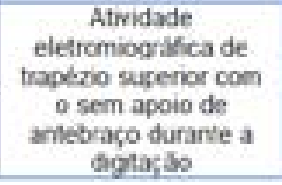 &  & 21 &  &  & 0 \\
\hline
\end{tabular}

\begin{tabular}{|c|c|c|c|c|c|c|c|}
\hline $\begin{array}{l}\text { MELLO } \\
\text { M.C.V.A et al. }\end{array}$ & 2017 &  &  & 43 &  &  & 0 \\
\hline $\begin{array}{l}\text { RONCATTO. } \\
\text { S.C. at al. }\end{array}$ & 2017 &  &  & 1 &  &  & 85 \\
\hline $\begin{array}{l}\text { SANTOS. } \\
\text { M.C.: } \\
\text { NRUEGER, E: } \\
\text { NEVES. E. }\end{array}$ & 2017 &  &  & 46 &  &  & B5 \\
\hline $\begin{array}{l}\text { DA sulva, } \\
\text { S.E.M, ot ot }\end{array}$ & $201 \%$ &  &  & 3 & EMG &  & BS \\
\hline
\end{tabular}

Fonte: Autores, 2018 
Tabela 2: Resumo dos estudos selecionados em relação às teses e dissertaç̃es.

\begin{tabular}{|c|c|c|c|c|c|c|c|c|}
\hline Avtor & Ans & Tinulo & osiotivo & Amostra & Natedo & Contribustä EVG & IES & $T / 0$ \\
\hline $\begin{array}{l}\text { MACALHAES, } \\
\text { K.C. }\end{array}$ & 2005 & 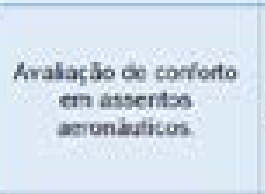 &  & 20 &  &  & ITA & D \\
\hline $\begin{array}{l}\text { PERROANO. } \\
\text { IS.V. }\end{array}$ & 2000 &  &  & 23 & Dhamometis e EMG &  & LEC & $T$ \\
\hline $\begin{array}{l}\text { SHIMANO } \\
\text { S.Q.N. }\end{array}$ & 2010 &  &  & 13 & $\begin{array}{l}\text { Antopometra } \\
\text { Ansise de Centadoso } \\
\text { eEMG }\end{array}$ & 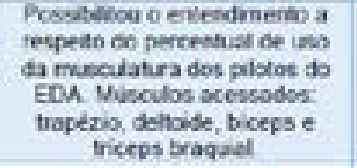 & USPFMRP & T \\
\hline ONETY, Q.C.s. & 2011 &  &  & 18 &  &  & USP & 0 \\
\hline DA SILVR, E.P. & 2011 &  &  & 4 &  &  & UFV & $\mathrm{T}$ \\
\hline $\begin{array}{l}\text { CUSTOOOO, } \\
\text { RAR. }\end{array}$ & 2011 &  &  & 10 & Dnammemera \& EUG &  & UNESP & T \\
\hline mus a & 2011 &  & 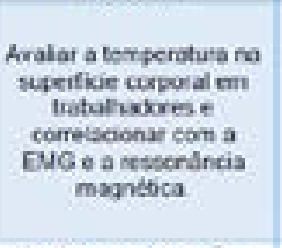 & is & 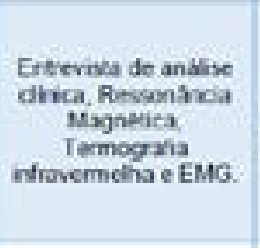 &  & UPPR & $\mathrm{T}$ \\
\hline LEONEL D.V. & 2012 &  &  & 18 &  &  & us? & $T$ \\
\hline
\end{tabular}

\begin{tabular}{|c|c|c|c|c|c|c|c|c|}
\hline SICVA LCC.B. & 2012 &  &  & ri &  &  & UFSCAR & T \\
\hline ERAOA O.W. & 2012 & 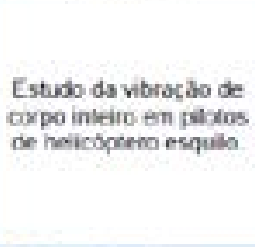 &  & 2 &  &  & uness & D \\
\hline BARBIERL D.F. & 2013 &  &  & so & hechoretra e EMG &  & UFSCAR & D \\
\hline SILVA, LG & 2014 &  &  & 28 &  & 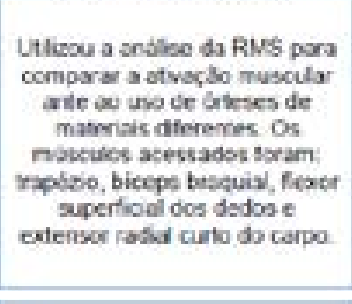 & UFSCAR & 0 \\
\hline $\begin{array}{l}\text { ROOPGQUES. } \\
\text { D.E. }\end{array}$ & 2014 &  &  & 7 & Dramometra e ENa &  & LNESP & D \\
\hline  & 2014 &  &  & $n$ &  &  & LNACiD & D \\
\hline OLVEIRA, $D . R$ & 204 &  & 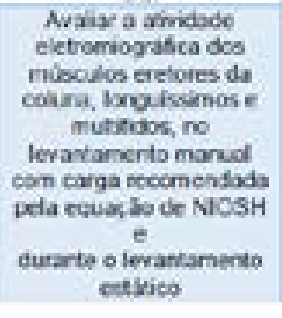 & 12 &  &  & UNESP & ${ }^{\top}$ \\
\hline
\end{tabular}




\begin{tabular}{|c|c|c|c|c|c|c|c|c|}
\hline $\begin{array}{l}\text { Macedo. } \\
\text { R.M.S. }\end{array}$ & 2014 &  &  & 35 &  &  & UTFPR & D \\
\hline NETO.LFN. & 2014 &  &  & 10. &  &  & FANERP & T \\
\hline $\begin{array}{l}\text { vontrelio, } \\
\text { J.AB. }\end{array}$ & 2015 &  & 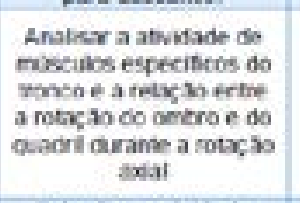 & ${ }^{3}$ & Creneera zo e EMac &  & unes: & o \\
\hline \multirow[t]{2}{*}{$\begin{array}{l}\text { GONCALLLES. } \\
\text { IS }\end{array}$} & 2015 &  &  & $23 e 15$ &  &  & UFSCAR & 0 \\
\hline & &  &  & & &  & & \\
\hline PINHEIRO, C.F. & 2018 &  &  & $\infty$ &  &  & USPIFMRP & D \\
\hline SANTOS. V.C. & 2016 &  &  & 44 &  &  & UTFPR & D \\
\hline \multirow[t]{2}{*}{$\begin{array}{c}\text { Paganello. } \\
\text { LR. }\end{array}$} & 2016 &  &  & 20 &  &  & UNESP & D \\
\hline & & & & & & 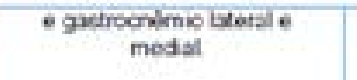 & & \\
\hline NOQUEIRA HC. & 2016 &  & 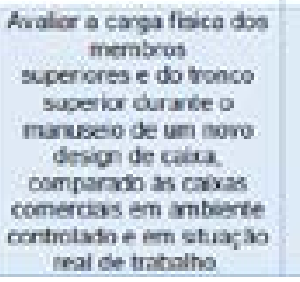 & 59 & 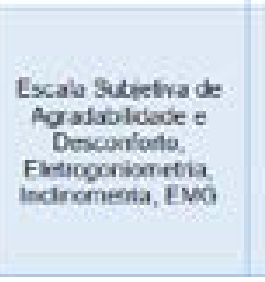 &  & UFSCAR & $T$ \\
\hline
\end{tabular}

\begin{tabular}{|c|c|c|c|c|c|c|c|c|}
\hline $\begin{array}{l}\text { VASCONSEL OS, } \\
\text { G.B.T. }\end{array}$ & 2017 &  &  & 6 &  &  & UFV & D \\
\hline \multirow[t]{2}{*}{ ARAUJO, N.C.K. } & 2017 &  &  & to &  &  & UFSCA & T \\
\hline & & & & &  & & & \\
\hline Kovino, cs.u. & 2017 & 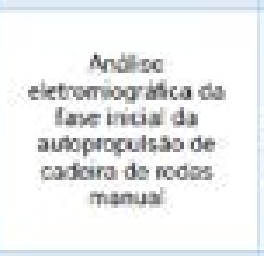 &  & 11 & EMS &  & usp & D \\
\hline ROCHA \&. & $201 \%$ &  &  & so &  &  & UNASC & D \\
\hline SILVA S.E.K. & 2018 & 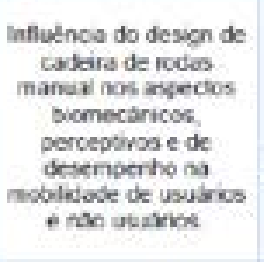 & 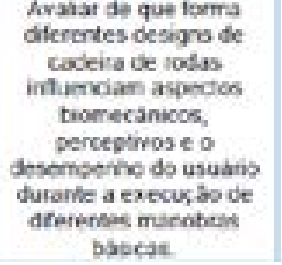 & 10 &  &  & UNESP & D \\
\hline
\end{tabular}

Fonte: Autores, 2018 
Eletromiografia em ergonomia no Brasil: Estado da arte. Uma revisão sistemática de literatura

Tabela 3: Artigo selecionado em anais de eventos.

\begin{tabular}{|c|c|c|c|c|c|c|}
\hline Autsores & Ane & Titulo & Objetives & Amostrs & Metoces & Contribuięas EVG \\
\hline $\begin{array}{c}\text { MERINO E.A.O. } \\
\text { ot al }\end{array}$ & 2017 & $\begin{array}{l}\text { Andise ergondmica e } \\
\text { biomecinica da coheia } \\
\text { da rair da mandoca. } \\
\text { estudo de caso. }\end{array}$ &  & 1 & $\begin{array}{c}\text { Dinamometris } \\
\text { Isomitsisa de Evtonsts } \\
\text { Lombar, EMC }\end{array}$ &  \\
\hline
\end{tabular}

Fonte: Autores, 2018 